\title{
Ethanol extract of Kalopanax septemlobus leaf induces caspase-dependent apoptosis associated with activation of AMPK in human hepatocellular carcinoma cells
}

\author{
CHEOL PARK $^{1^{*}}$, JI-SUK JEONG $^{2 *}$, JIN-WOO JEONG $^{3}$, YONG-JOO KIM ${ }^{3}$, YEON-KWON JUNG ${ }^{4}$, GEUN-BAE GO $^{4}$, \\ SUNG OK KIM ${ }^{5}$, GI-YOUNG KIM ${ }^{6}$, SU-HYUN HONG ${ }^{7}$, YOUNG HYUN YOO ${ }^{8}$ and YUNG HYUN CHOI $^{2,7}$ \\ ${ }^{1}$ Department of Molecular Biology, College of Natural Sciences and Human Ecology, ${ }^{2}$ Anti-Aging Research Center, \\ Dongeui University, Busan 614-714; ${ }^{3}$ Gurye Wild Flower Institute; ${ }^{4}$ Gurye-gun Agricultural Center, Gurye 504-805; \\ ${ }^{5}$ Department of Food Science and Biotechnology, College of Engineering, Kyungsung University, Busan 608-736; \\ ${ }^{6}$ Laboratory of Immunobiology, Department of Marine Life Sciences, Jeju National University, Jeju 690-756; \\ ${ }^{7}$ Department of Biochemistry, Dongeui University College of Korean Medicine, Busan 614-714; \\ ${ }^{8}$ Department of Anatomy and Cell Biology, Dong-A University College of Medicine \\ and Mitochondria Hub Regulation Center, Busan 602-714, Republic of Korea
}

Received August 26, 2015; Accepted October 21, 2015

DOI: $10.3892 /$ ijo.2015.3233

\begin{abstract}
The Kalopanax septemlobus leaf (Thunb.) Koidz. has been used as a traditional medicine herb for the treatment of various human diseases for hundreds of years. In this study, we investigated the mechanism underlying the inhibitory effects of an ethanol extract of K. septemlobus leaf (EEKS) on proliferation of HepG2 hepatocellular carcinoma cells. For this study, cell viability and apoptosis were evaluated using the MTT [3-(4,5-dimethylthiazol-2-yl)-2,5-diphenyltetrazolium bromide] assay, DAPI (4,6-diamidino-2-phenylindole) staining, agarose gel electrophoresis, and flow cytometry. Measurements of the mitochondrial membrane potential (MMP), caspase activity assays and western blots were conducted to determine whether HepG2 cell death occurred by apoptosis. Treatment of HepG2 cells with EEKS concentration-dependently reduced cell survival while significantly increasing the ratio of apoptotic cells. EEKS treatment increased the levels of the death receptors (DRs), DR4 and DR5, and activated caspases, as well
\end{abstract}

Correspondence to: Professor Yung Hyun Choi, Department of Biochemistry, Dongeui University College of Korean Medicine, 52-57 Yangjeong-ro, Busanjin, Busan 614-052, Republic of Korea

E-mail: choiyh@deu.ac.kr

Professor Young Hyun Yoo, Department of Anatomy and Cell Biology, Dong-A University College of Medicine and Mitochondria Hub Regulation Center, Busan 602-714, Republic of Korea

E-mail: yhyoo@dau.ac.kr

*Contributed equally

Key words: Kalopanax septemlobus, hepatocellular carcinoma, apoptosis, caspase, AMP-activated protein kinase as promoting proteolytic degradation of poly(ADP-ribose)polymerase associated with the downregulation of protein expression of members of the inhibitor of apoptosis protein family. Treatment with EEKS also caused truncation of Bid, translocation of pro-apoptotic Bax to the mitochondria, and loss of mitochondrial membrane permeabilization, thereby inducing the release of cytochrome $c$ into the cytosol. However, treatment of HepG2 cells with a pan-caspase inhibitor reversed EEKS-induced apoptosis and growth suppression, indicating that EEKS appears to induce apoptosis though a caspase-dependent mechanism involving both intrinsic and extrinsic apoptotic pathways. In addition, the phosphorylation level of AMP-activated protein kinase (AMPK) was elevated when cells were exposed to EEKS. A specific inhibitor for AMPK attenuated the EEKS-induced activation of caspases, and consequently prevented the EEKS-induced apoptosis and reduction in cell viability. Overall, our findings suggest that EEKS inhibits the growth of HepG2 cells by inducing AMPK-mediated caspase-dependent apoptosis, suggesting the potential therapeutic application of EEKS in the treatment or prevention of cancers.

\section{Introduction}

Hepatocellular carcinoma (HCC) is one of the most common and lethal cancers in the human population, ranking as the third most common cause of cancer-associated mortality worldwide $(1,2)$. Persistent hepatitis B virus infection is a well-established risk factor for liver cancer, but HCC is caused by a complex interaction between numerous factors, such as environmental exposure, extensive alcohol intake, transgenic oncogenes, and other causes of hepatic cirrhosis (3-6). Despite recent progress in anticancer therapy, the efficacy of currently available anticancer treatments is limited because of natural or acquired resistance to conventional chemotherapy and limited 
efficacy of radiotherapy (7). Patients with advanced HCC therefore continue to show poor outcomes $(8,9)$, which emphasizes the need to identify and develop more potent anticancer drugs with increased selectivity and reduced toxicity for HCC treatment.

Apoptosis is a highly regulated process of programmed cell death, and disruption of this process represents a major contributing factor in the pathology of cancer (10). In general, apoptosis may be initiated through two major pathways, the extrinsic [death receptor (DR)-mediated] and the intrinsic (mitochondria-mediated) pathways $(11,12)$. The extrinsic pathway is initiated by binding of death ligands with their cognate receptors, which leads to the activation of caspase- 8 , while the intrinsic pathway is initiated by release of cytochrome $c$ from mitochondria into the cytosol following loss of inner mitochondrial membrane integrity and activation of caspase-9 $(11,13)$. A series of signaling cascades is activated, and both pathways ultimately result in activation of caspase-3 and -7 , the final executioners of apoptotic cell death $(14,15)$. Therefore, agents that target the apoptosis pathway without affecting normal cells have significant potential as drug targets for cancer treatment.

Accumulating evidence now indicates that cancer metabolism mediates cancer development and progression via its activation of related signaling pathways, such as the AMP-activated protein kinase (AMPK) pathway $(16,17)$. Under metabolic stress conditions, where decreases in intracellular ATP and increases in AMP levels are common, AMPK is activated by phosphorylation of a critical amino acid residue (Thr172) to function as a major metabolic switch that maintains energy homeostasis $(18,19)$. Once activated, AMPK suppresses cell proliferation in tumor as well as non-tumor cells, through regulation of the cell cycle, apoptosis, autophagy, and inhibition of protein synthesis (20-25). Current evidence supports the alteration of AMPK levels in various cancers, implicating AMPK as a potential target for prevention and/or treatment of cancer, specifically HCC (26-28). Several studies have also demonstrated that activation of AMPK induces apoptosis in many human cancer cells, including HCC cells (29-31). This finding indicates that AMPK plays a critical role in HCC and that its loss can contribute to the progression of HCC. Indeed, a variety of AMPK activators show anticancer effects $(27,32,33)$. For example, metformin, a common diabetes drug, shows anticancer effects against HCC by activation of AMPK (27,34-36). Similarly, a number of medicinal herb extracts exert anticancer effects through activation of AMPK-dependent cell death pathways $(29,31,37-40)$.

Recently, interest has been increasing in traditional herbs, with pharmacological activity confirmed by traditional medicine, as new therapies for diseases like cancer that are difficult to treat $(41,42)$. One of these is the deciduous tree, Kalopanax septemlobus (Thunb.) Koidz. (Araliacieae; common name: prickly castor oil tree), which is widely recognized in its native region of northeastern Asia as a treatment for rheumatic arthritis, nephritis edema, cholera, dysentery, and neurotic pain. The extracts and/or components that $K$. septemlobus possess have been confirmed recently to have pharmacological activities ranging from anti-oxidant, anti-inflammatory, and hypoglycemic functions to regulation of neurite outgrowth (43-46). The molecular mechanisms underlying the effects of
K. septemlobus extracts appear to involve apoptosis, but the precise pathways have not been elucidated to date. In the present study, we evaluated whether an ethanol extract of $K$. septemlobus leaves (EEKS) could inhibit the growth of HepG2 human HCC cells. We found that EEKS triggered caspasedependent apoptosis through activation of both intrinsic and extrinsic pathways. We also investigated the potential roles for and underlying mechanisms of AMPK signaling in mediating EEKS-induced apoptosis in HepG2 cells.

\section{Materials and methods}

Preparation of EEKS. The leaves of K. septemlobus were obtained from Gurye Wild Flower Institute (Gurye, Korea) and authenticated by Professor S.H. Hong, Department of Biochemistry, Dongeui University College of Korean Medicine (Busan, Korea). The dried leaves (50 $\mu \mathrm{g}$ ) were cut into small pieces, ground into a fine powder, and then soaked with $500 \mathrm{ml} \mathrm{70 \%} \mathrm{ethanol}(500 \mathrm{ml})$ for 2 days. The extracted liquid was filtered twice through Whatman No. 3 filter paper to remove any insoluble materials and was then concentrated using a rotary evaporator (Rikakikai Co., Tokyo, Japan). The resulting extract (EEKS) was dissolved in dimethylsulfoxide (DMSO; Sigma-Aldrich Co., St. Louis, MO, USA) to a final concentration of $200 \mathrm{mg} / \mathrm{ml}$ (extract stock solution) and was subsequently diluted with medium to the desired concentration prior to use.

Cell culture. HepG2 HCC cells and Chang liver cells (an immortalized non-tumor cell line derived from normal liver tissue) were purchased from the American Type Culture Collection (Manassas, MD, USA). The cells were cultured at $37^{\circ} \mathrm{C}$ in humidified air with $5 \% \mathrm{CO}_{2}$ in RPMI-1640 medium (Gibco-BRL, Gaithersburg, MD, USA), supplemented with $10 \%$ fetal bovine serum (FBS, Gibco-BRL), $100 \mathrm{U} / \mathrm{ml}$ of penicillin, and $100 \mathrm{mg} / \mathrm{ml}$ of streptomycin (Sigma-Aldrich Co.), with or without added EEKS.

Cell viability assay. Cells were incubated with EEKS, with or without z-Val-Ala-Asp-fluoromethylketone (z-VAD-fmk, a caspase-3 inhibitor; Calbiochem, San Diego, CA, USA) or dorsomorphin dihydrochloride (compound C, an AMPK inhibitor; Tocris Bioscience, Bristol, UK) at serial concentrations. The culture medium was then aspirated and the cells were washed with phosphate-buffered saline (PBS), followed by incubation with $0.5 \mathrm{mg} / \mathrm{ml}$ of 3-[4,5-dimethylthiazol2-yl]-2,5-diphenyltetrazolium bromide (MTT, Sigma-Aldrich Co.) at $37^{\circ} \mathrm{C}$ for $2 \mathrm{~h}$. After incubation, the supernatant was removed and the cells were treated with DMSO to dissolve the formazan reaction product. The concentration of formazan was determined by measuring the absorbance at $540 \mathrm{~nm}$ using an enzyme-linked immunosorbent assay (ELISA) reader (Molecular Devices, Sunnyvale, CA, USA).

Fluorescence microscopy examination of apoptosis. Apoptosis was assessed by washing the cells with PBS, followed by fixation in 3.7\% paraformaldehyde (Sigma-Aldrich Co.) in PBS for $10 \mathrm{~min}$ at room temperature. The fixed cells were washed, permeabilized with $0.2 \%$ Triton X-100 in PBS for $10 \mathrm{~min}$ at room temperature, and then incubated with $2.5 \mu \mathrm{g} / \mathrm{ml}$ 
4,6-diamidino-2-phenylindole (DAPI, Sigma-Aldrich Co.) for $10 \mathrm{~min}$ at room temperature. The cells were washed twice with PBS and images were then captured using a fluorescence microscope (Carl Zeiss, Jena, Germany).

Detection of apoptosis by DNA laddering assay. The control and EEKS-treated cells were harvested and washed with PBS, and the pellets were lysed for $1 \mathrm{~h}$ on ice in lysis buffer containing $10 \mathrm{mM}$ Tris- $\mathrm{HCl}$ (pH 7.4), $150 \mathrm{mM} \mathrm{NaCl}, 5 \mathrm{mM}$ trypsin-ethylenediaminetetraacetic acid (EDTA), and $0.5 \%$ Triton X-100. Following centrifugation at 19,000 x g for $30 \mathrm{~min}$ at $4^{\circ} \mathrm{C}$, DNA in the supernatant was extracted in an equal volume of neutral phenol :chloroform :isoamylalcohol (25:24:1, v/v/v, Sigma-Aldrich Co.). Equal volumes of DNA samples were then separated by electrophoresis in $1.5 \%$ agarose gel at $40 \mathrm{~V}$, stained with $0.1 \mu \mathrm{g} / \mathrm{ml}$ ethidium bromide (EtBr, SigmaAldrich Co.), and visualized under ultraviolet light.

DNA flow cytometric detection of apoptosis. After treatment with EEKS, the cells were harvested, washed twice with ice-cold PBS, and fixed with $75 \%$ ethanol at $4^{\circ} \mathrm{C}$ for $30 \mathrm{~min}$. The cells were then stained with $5 \mu$ l Annexin V-fluorescein isothiocyanate (FITC) (R\&D Systems, Minneapolis, MN, USA) and $5 \mu \mathrm{l}$ propidium iodide (PI, Sigma-Aldrich Co.). The samples were incubated for $15 \mathrm{~min}$ at room temperature in the dark, and then the degree of apoptosis was quantified by flow cytometry as a percentage of the Annexin V-positive and PI-negative (Annexin $\mathrm{V}^{+} / \mathrm{PI}^{-}$cells) cells (Becton-Dickinson, San Jose, CA, USA).

Protein extraction and western blot analysis. After treatment with different concentrations of EEKS, the cells were lysed in buffer containing $40 \mathrm{mM}$ Tris ( $\mathrm{pH} 8.0$ ), $120 \mathrm{mM} \mathrm{NaCl}$, $0.5 \%$ Nonidet P-40, $0.1 \mathrm{mM}$ sodium orthovanadate, $2 \mu \mathrm{g} / \mathrm{ml}$ aprotinin, $2 \mu \mathrm{g} / \mathrm{ml}$ leupeptin and $100 \mu \mathrm{g} / \mathrm{ml}$ phenymethylsulfonyl fluoride. In a parallel experiment, the mitochondrial and cytosolic fractions were isolated using a mitochondrial fractionation kit (Active Motif, Carlsbad, CA, USA) according to the manufacturer's instructions. The Bio-Rad protein assay (Bio-Rad, Hercules, CA, USA) was used according to the manufacturer's instructions to determine the protein concentrations. For western blot assays, equal amounts of protein (30-50 $\mu \mathrm{g} /$ lane) were subjected to electrophoresis on sodium dodecyl sulfate (SDS)-polyacrylamide gels, and then transferred onto nitrocellulose membranes (Schleicher and Schuell, Keene, NH, USA). The membranes were blocked with Tris-buffered saline (10 mM Tris- $\mathrm{Cl}, \mathrm{pH}$ 7.4) containing $0.5 \%$ Tween-20 and 5\% non-fat dry milk for $1 \mathrm{~h}$ at room temperature and incubated with the primary antibodies overnight. Membranes were then washed with PBS and incubated with the secondary antibody conjugated to horseradish peroxidase (Amersham Co., Arlington Heights, IL, USA) for $1 \mathrm{~h}$ at room temperature. Immunoreactivity was detected by using the enhanced chemiluminescence (ECL) western blotting detection system (Amersham Co.) according to the manufacturer's instructions. The primary antibodies used in this study were as follows: actin $(1: 1,000$, sc-7120; rabbit polyclonal, Santa Cruz Biotechnology, Inc., Santa Cruz, CA, USA), caspase-3 (1:1,000, sc-7272; mouse monoclonal, Santa Cruz Biotechnology, Inc.), caspase-8 (1:1,000, sc-7890; rabbit polyclonal, Santa Cruz Biotechnology, Inc.), caspase-9 (1:1,000, sc-7885; rabbit polyclonal, Santa Cruz Biotechnology, Inc.), PARP (1:1,000, sc-7150; rabbit polyclonal, Santa Cruz Biotechnology, Inc.), XIAP (1:1,000, sc-11426; rabbit polyclonal, Santa Cruz Biotechnology, Inc.), cIAP-1 (1:1,000, sc-7943; rabbit polyclonal, Santa Cruz Biotechnology, Inc.), cIAP-2 (1:1,000, sc-7944; rabbit polyclonal, Santa Cruz Biotechnology, Inc.), TRAIL (1:500, sc-7877; rabbit polyclonal, Santa Cruz Biotechnology, Inc.), DR4 (1:1,000, sc-7863; rabbit polyclonal, Santa Cruz Biotechnology, Inc.), DR5 (1:1,000, sc-65314; mouse monoclonal, Santa Cruz Biotechnology, Inc.), Fas (1:1,000, sc-715; rabbit polyclonal, Santa Cruz Biotechnology, Inc.), FasL (1:1,000, sc-957; rabbit polyclonal, Santa Cruz Biotechnology, Inc.), Bcl-2 (1:1000, sc-509; mouse monoclonal, Santa Cruz Biotechnology, Inc.), Bax (1:1,000, sc-493; rabbit polyclonal, Santa Cruz Biotechnology, Inc.), Bid (1:500, sc-11423; rabbit polyclonal, Santa Cruz Biotechnology, Inc.), cytochrome $c$ (1:500, sc-7159; rabbit polyclonal, Santa Cruz Biotechnology, Inc.), COX IV (1:1,000, sc-376731; mouse monoclonal, Santa Cruz Biotechnology, Inc.), AMPK (1:1,000, sc-25792; rabbit polyclonal, Santa Cruz Biotechnology, Inc.), p-AMPK (1:500, 2535; rabbit polyclonal, Cell Signaling Technology, Inc., Boston, MA, USA), ACC (1:1,000, sc-30212; rabbit polyclonal, Santa Cruz Biotechnology, Inc.) and p-ACC (1:500, 3661; rabbit polyclonal, Cell Signaling Technology, Inc.)

Measurement of mitochondrial membrane potential $(M M P, \Delta \psi m)$. The MMP values were determined using the dual-emission potential-sensitive probe, 5,5',6,6'-tetrachloro-1,1',3,3'-tetraethyl-imidacarbocyanine iodide (JC-1, Sigma-Aldrich Co.), which is internalized and concentrated by respiring mitochondria and can therefore reflect MMP changes in cells. Briefly, cells were fixed and permeabilized with $0.2 \%$ Triton $\mathrm{X}-100$ in PBS for $10 \mathrm{~min}$ at room temperature, and then incubated with $10 \mu \mathrm{M} \mathrm{JC}-1$ for $30 \mathrm{~min}$ at $37^{\circ} \mathrm{C}$ in the dark. Subsequently, the cells were washed with PBS to remove unbound dye, and the amount of JC-1 retained by 10,000 cells per sample was measured at 488 and $575 \mathrm{~nm}$ using a flow cytometer.

Caspase activity assay. The activities of the caspases were assessed by marking cells with colorimetric assay kits (R\&D Systems), which utilize synthetic tetrapeptides (Asp-GluVal-Asp (DEAD) for caspase-3; Ile-Glu-Thr-Asp (IETD) for caspase-8; and Leu-Glu-His-Asp (LEHD) for caspase-9) labeled with p-nitroaniline (pNA) that is linked to the end of the caspase-specific substrate. Briefly, the cells were washed with PBS and lysed in the supplied lysis buffer, according to the manufacturer's instructions. The supernatants were collected and incubated at $37^{\circ} \mathrm{C}$ for $2 \mathrm{~h}$ in the dark with the supplied reaction buffer containing dithiothreitol and DEAD-pNA, IETD-pNA, or LEHD-pNA as substrates. The reactions were measured by changes in absorbance at $405 \mathrm{~nm}$ using an ELISA reader.

Statistical analysis of data. The experiments were repeated three times, and the results were expressed as means \pm standard deviation (SD). A one-way analysis of variance (ANOVA), followed by Dunnett's t-test, was applied to assess the statis- 


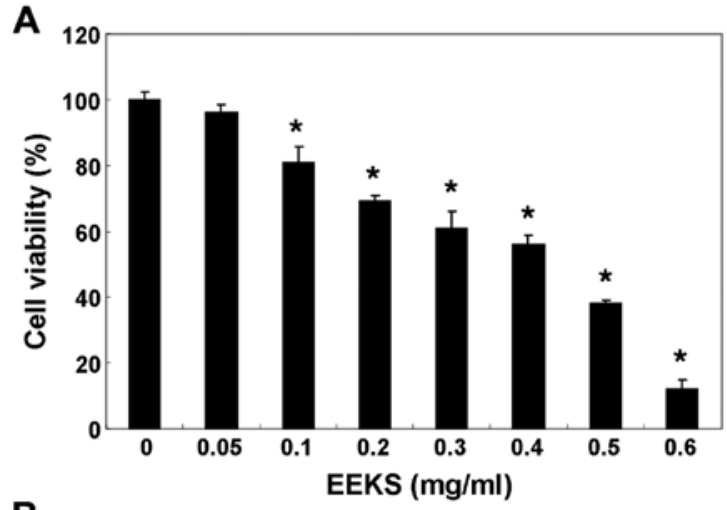

B

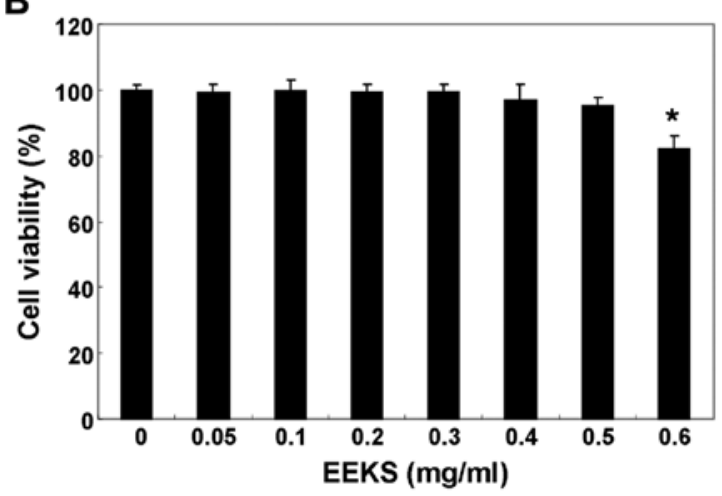

Figure 1. Inhibition of cell viability by EEKS treatment of HepG2 cells. HepG2 (A) and Chang liver (B) cells were treated with various concentrations of EEKS for $24 \mathrm{~h}$. Cell viability was determined by the MTT assay. Statistical significance was determined using a one-way ANOVA ( $\mathrm{p}<0.05$ vs. untreated control). tical significance of the difference among the study groups. A value of $\mathrm{p}<0.05$ was considered to be statistically significant.

\section{Results}

EEKS treatment suppresses the viability of Hep 22 cells. The effects of EEKS on cell viability were evaluated by stimulating the HepG2 cells with various concentrations of EEKS for $24 \mathrm{~h}$, and performing an MTT assay. As shown in Fig. 1A, EEKS caused significant concentration-dependent decreases in cell viability in HepG2 cells. The results of an additional experiment using Chang liver cells, conducted to examine the effect of EEKS on the proliferation of normal cells, are shown in Fig. 1B. EEKS concentrations $\leq 500 \mu \mathrm{mg} / \mathrm{ml}$ did not induce cytotoxicity, whereas $600 \mu \mathrm{g} / \mathrm{ml}$ EEKS significantly reduced cell viability. Therefore, in this study, conditions for subsequent experiments were chosen that induced no cytotoxicity in Chang liver cells.

EEKS treatment enhances apoptosis in HepG2 cells. The possibility that the growth inhibitory activity of EEKS was caused by an induction of apoptosis was examined based on three established criteria for assessment of apoptosis. First, morphological changes in HepG2 cells were determined using DAPI staining, as shown in Fig. 2A. A significant number of cells showed chromatin condensation, loss of nuclear construction, and formation of apoptotic bodies following EEKS treatment, whereas these features were not observed in control cells. Second, treatment with EEKS induced progressive accu-
A

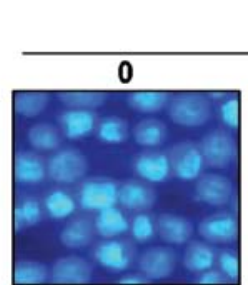

0.3

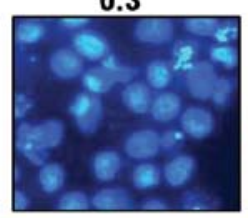

$\operatorname{EEKS~(mg/ml)~}$ 0.1

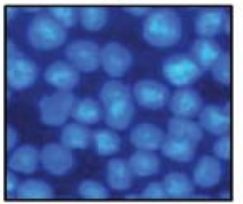

0.4

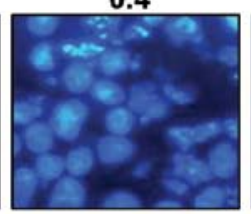
0.2

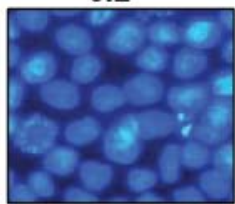

0.5

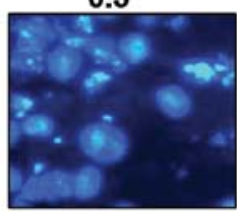

B



C

EEKS $(\mathrm{mg} / \mathrm{ml})$

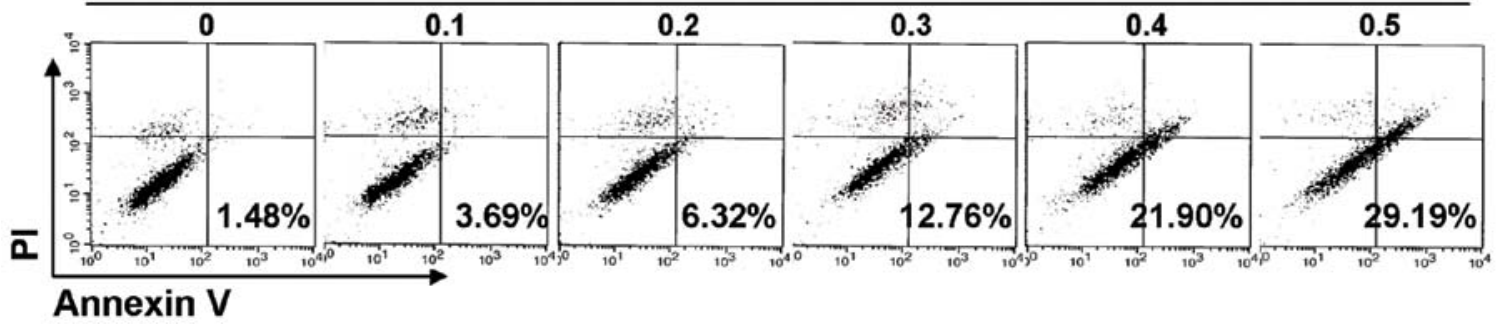

Figure 2. Induction of apoptosis by EEKS treatment in HepG2 cells. The cells were treated for $24 \mathrm{~h}$ with the indicated concentrations of EEKS. (A) Cells were fixed and stained with DAPI to visualize DNA. The stained nuclei were then observed with a fluorescence microscope (original magnification, $\mathrm{x} 400$ ). (B) DNA fragmentation was analyzed by extracting genomic DNA, electrophoresis in a $1.5 \%$ agarose gel, and then visualization by EtBr staining. (C) The degree of apoptosis induced by EEKS was quantified by staining the cells with FITC-conjugated Annexin V and PI for flow cytometry analysis. The percentages of apoptotic cells were determined by counting the percentage of Annexin V-positive cells. Each point represents the means of two independent experiments. 
A

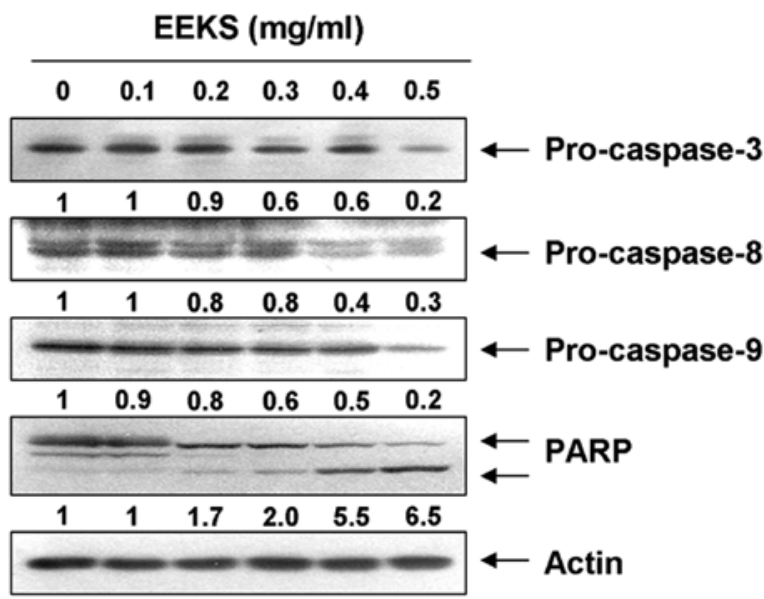

B

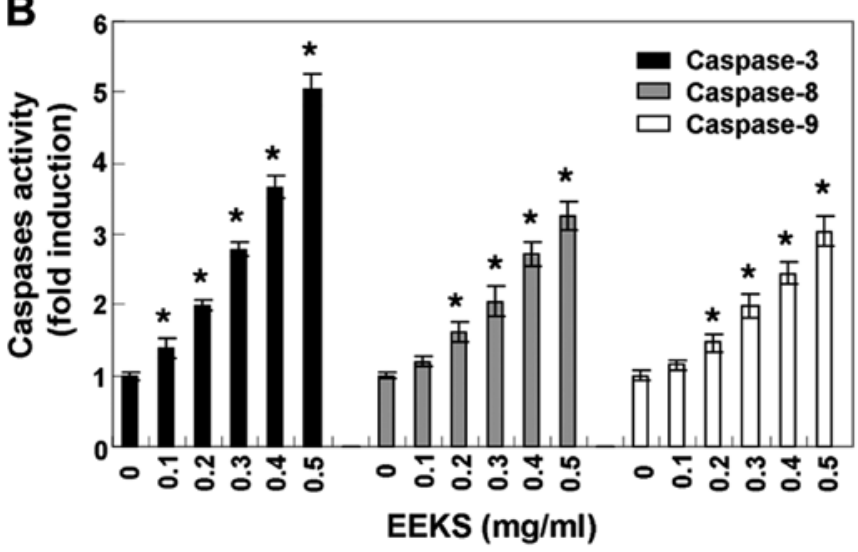

Figure 3. Activation of caspases and degradation of PARP by EEKS treatment in HepG2 cells. The cells were treated with the indicated concentrations of EEKS for $24 \mathrm{~h}$. (A) The cells were lysed and cellular proteins were separated by SDS-polyacrylamide gel electrophoresis and then transferred to nitrocellulose membranes. The membranes were probed with the indicated antibodies. Proteins were visualized using an enhanced chemiluminescence (ECL) detection system. Equal loading was confirmed using actin as an internal control. The relative ratios of expression in the results of western blotting were presented at the bottom of each of the results as relative values of actin expression. (B) The cells were lysed and aliquots (50 $\mu \mathrm{g}$ of protein) were assayed for caspase- $3,-8$, and -9 activity using DEVD-pNA, IETD-pNA, and LEHD-pNA, respectively, as substrates. The fluorescent products released were measured. Statistical significance was determined using a one-way ANOVA ("p<0.05 vs. untreated control).

mulation of fragmented DNA in a concentration-dependent manner, which appeared as a typical ladder pattern of DNA fragmentation, another hallmark of apoptosis (Fig. 2B). In addition, flow cytometry analysis confirmed an increased accumulation of Annexin V-positive cells following EEKS treatment, indicating a greater degree of apoptosis in cells treated with EEKS (Fig. 2C). These results suggested that EEKS-induced apoptosis in HepG cells contributed to its antiproliferative and cytotoxic effects.

EEKS-induced apoptosis is caspase-dependent in HepG2 cells. The molecular mechanism of EEKS-induced apoptosis in HepG2 cells was examined by determining whether EEKS affected activation of caspase cascades - key executioners of apoptosis. Western blot analyses revealed that the expression levels of pro-caspase- 8 and -9 , upstream activators of caspase-3/-7 in the extrinsic and intrinsic pathways, respectively, were downregulated in a concentration-dependent manner (Fig. 3A). In addition to the reduced expression of pro-caspase- 8 and -9 , the level of pro-caspase-3 was also concentration-dependently reduced in response to EEKS treatment, and correspondingly, EEKS treatment led to progressive proteolytic cleavage of PARP (116-kDa), a known caspase-3 substrate (47), to yield an $85-\mathrm{kDa}$ cleaved fragment. In addition, equal amounts of proteins from lysates from cells treated with EEKS were assayed to assess in vitro caspase activity. As shown in Fig. 3B, treatment with EEKS significantly increased the activity of caspase $-3,-8$ and -9 .

We further confirmed the involvement of activation of caspases in the apoptosis induced by EEKS by pretreating the cells with a pan-caspase inhibitor, z-VAD-fmk, for $1 \mathrm{~h}$, followed by EEKS treatment. As shown in Fig. 4A and B, pre-treatment with $\mathrm{z}$-VED-fmk significantly prevented the appearance of apoptotic features such as chromatin condensation and DNA fragmentation. Flow cytometry analysis and MTT assay also revealed that the pan-caspase inhibitor suppressed EEKS-induced apoptosis and reduction in viability in HepG2 cells (Fig. 4C and D). These results clearly indicate that EEKS-triggered apoptosis may be mediated through caspase-dependent extrinsic and intrinsic pathways in HepG2 cells. In addition, EEKS treatment reduced the expression of members of the inhibitor of the apoptosis proteins (IAP) family, such as XIAP, cIAP-1, and cIAP-2 (Fig. 5), which bind to caspases and lead to their inactivation $(48,49)$. This finding indicated that EEKS-induced activation of caspases was associated with a reduction in IAP family proteins.

EEKS treatment induces the expression of death receptor (DR)-related proteins and truncation of Bid in HepG2 cells. We further explored the induction of apoptosis of HepG2 cells by EEKS by detecting the expression levels of DR-mediated apoptosis regulators and $\mathrm{Bid}$, a $\mathrm{BH} 3$ pro-apoptotic protein, which might be involved in the regulation of extrinsic apoptosis pathway. Western blot analysis revealed that the levels of DR ligands, such as the tumor necrosis factor-related apoptosis-inducing ligand (TRAIL) and Fas ligand (FasL), were relatively unchanged in response to EEKS treatment; however, the levels of DR4 and DR5, but not Fas, increased markedly in a concentration-dependent manner (Fig. 3). A subsequent immunoblotting analysis revealed that exposure of HepG2 cells to EEKS caused a progressive accumulation of truncated Bid (tBid) (Fig. 4A), presumably resulting from truncation by activated caspase- 8 . These results indicate that the cytotoxic effects induced by EEKS could be mediated through DR-mediated apoptosis, thereby accentuating crosstalk between the intrinsic and extrinsic apoptosis pathways.

EEKS treatment activates the mitochondria-mediated intrinsic apoptosis pathway in HepG2 cells. We next evaluated the effect of EEKS on the mitochondrial apoptosis signaling pathway. Notably, the total levels of pro-apoptotic Bax and anti-apoptotic Bcl-2 proteins remained unchanged in response to EEKS treatment (Fig. 6A); however, EEKS treatment decreased the cytosolic levels of Bax, whereas its mitochondrial levels were increased significantly after treatment with increasing concentrations of EEKS (Fig. 6B). An indicative 
A
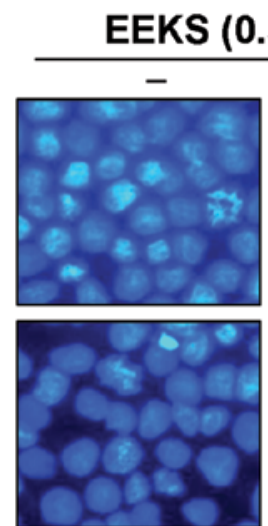

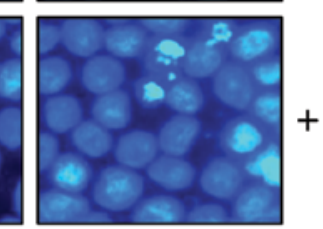

C

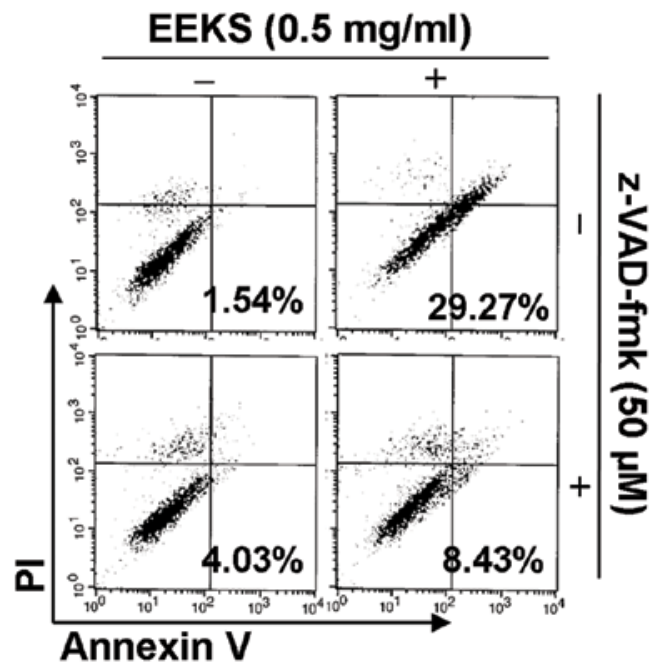

B

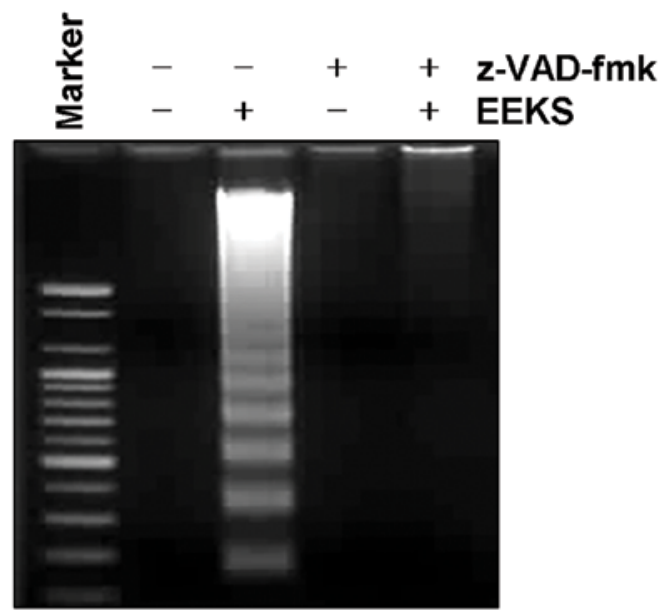

D

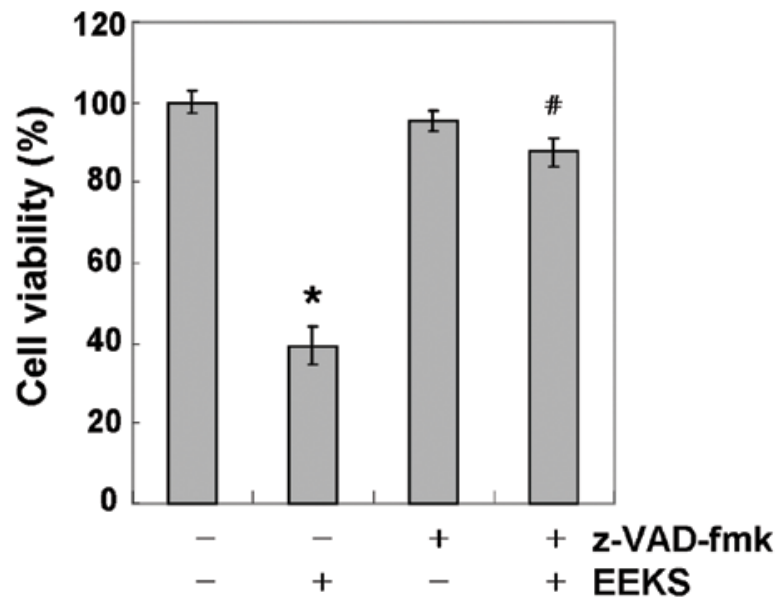

Figure 4. Inhibition of EEKS-induced apoptosis in HepG2 cells by a pan-caspase inhibitor. The cells were pre-treated for $1 \mathrm{~h}$ with or without $\mathrm{z}$-VED-fmk $(50 \mu \mathrm{M})$, and then treated with EEKS $(0.5 \mathrm{mg} / \mathrm{ml})$ for an additional $24 \mathrm{~h}$. (A) The cells were stained with DAPI and observed with a fluorescence microscope (original magnification, $\mathrm{x} 400$ ). (B) The genomic DNA from the cells was extracted, separated by electrophoresis in a 1.5\% agarose gel, and then visualized under UV light after staining with EtBr. (C) The percentage of apoptotic cells (Annexin $\mathrm{V}^{+}$cells) was analyzed using flow cytometry analysis. The data are the means of the two different experiments. (D) Cell viability was determined by the MTT assay. Each point represents the mean \pm SD of three independent experiments ( ${ }^{*} \mathrm{p}<0.05$ vs. untreated control; ${ }^{\#} \mathrm{p}<0.05$ vs. EEKS-treated cells).

stage in the intrinsic apoptotic pathway is the depolarization of the mitochondrial membrane and the subsequent increase in permeability of the outer membrane, followed by pore formation and the release of proapoptotic molecules including cytochrome $c$ into cytosol $(50,51)$. The present results demonstrated that treatment with increasing concentrations of EEKS significantly reduced the MMP levels (Fig. 6C), suggesting depolarization of the mitochondria by EEKS. Further characterization of the mitochondrial-mediated apoptotic effect of EEKS, by analyzing the release of cytochrome $c$, revealed that EEKS treatment promoted a concentration-dependent increase in the release of cytochrome $c$ from mitochondria into the cytosol (Fig. 4B), suggesting that EEKS also activated the mitochondria-mediated intrinsic apoptosis pathway in HepG2 cells.

EEKS treatment induces apoptosis and lowers cell viability of HepG2 cells via AMPK activation. Several reports have demonstrated that activation of AMPK leads to the induction of apoptosis in numerous human cancer cell types (29-31). We therefore investigated whether the phosphorylation of AMPK is induced by EEKS. As demonstrated in Fig. 7A, when compared with the basal level, EEKS treatment led to increased levels of phosphorylation of AMPK (Thr172) in a concentration-dependent manner, without inducing significant changes in the total protein levels. Consistent with the activation of AMPK, the phosphorylation of acetyl-CoA carboxylase (ACC) at Ser79 (the best-characterized site phosphorylated by AMPK) (52) also increased after EEKS administration, indicating that the AMPK pathway in HepG2 cells was activated in the presence of EEKS.

We further confirmed the relationship between apoptosis induction and AMPK activation in EEKS-stimulated HepG2 cells by examining the effects of compound $\mathrm{C}$, a specific AMPK inhibitor. As shown in Fig. 7B, treatment with compound $\mathrm{C}$ blocked phosphorylation of AMPK as well as ACC and, inter- 


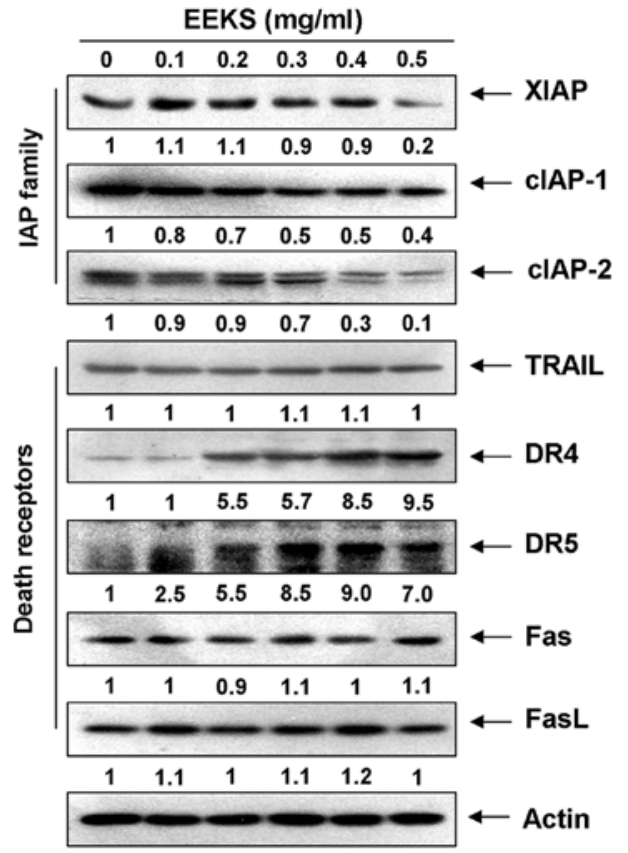

Figure 5. Effects of EEKS treatment on the levels of IAP family and DR-related IAP family proteins in HepG2 cells. Cells were incubated with various concentrations of EEKS for $24 \mathrm{~h}$, and then the cellular proteins were separated by SDS-polyacrylamide gel electrophoresis and transferred to nitrocellulose membranes. The membranes were probed with the indicated antibodies, and the proteins were visualized using an enhanced chemiluminescence (ECL) detection system. Actin was used as an internal control. The relative ratios of expression in the results of western blotting are presented at the bottom of each result as relative value of actin expression. estingly, prevented cleavage of caspases (caspase-3, -8 and -9) and degradation of PARP in EEKS-treated HepG2 cells, implying a linkage between caspase activation and AMPK activation. In line with these observations, results in Fig. 8 demonstrated that AMPK inhibition significantly reduced the increase in apoptosis induced by EEKS (Fig. 8A-C) and the cell viability loss (Fig. 8D). These results suggested that EEKS-induced apoptosis and loss of viability were mediated by activation of AMPK, and that AMPK was probably upstream of caspase activation in the signaling pathway involved in this process.

\section{Discussion}

Apoptosis is an essential biological process for maintaining cell stability, structure, and function, and for proper development. Therefore, induction of apoptosis in target cells is an ideal strategy for anticancer therapy. In this process, caspase- and mitochondria-mediated apoptosis coordinately occur in response to a wide range of death stimuli $(15,53)$. The caspase-8-mediated extrinsic apoptotic pathway and the tBidmediated intrinsic apoptotic pathway can both contribute, in widely varying degrees, to death receptor-induced apoptosis $(11,54)$. Our data revealed that EEKS promoted the expression of DR4 and DR5, activation of caspase-8 and -3, and concomitant PARP cleavage, together with a rise in tBid. Therefore, the data indicate that the extrinsic pathway contributes, at least
A

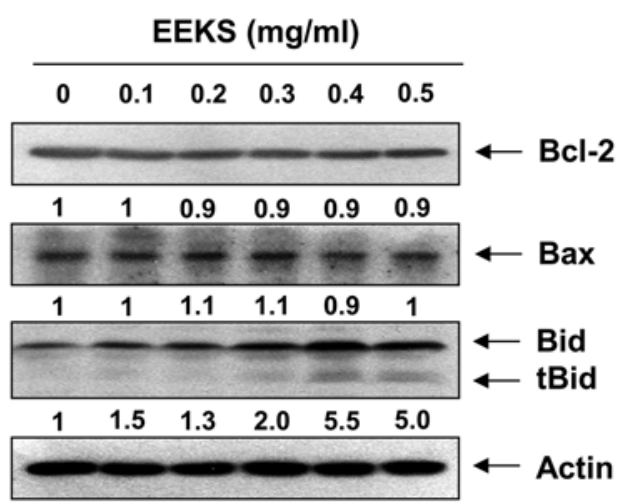

B

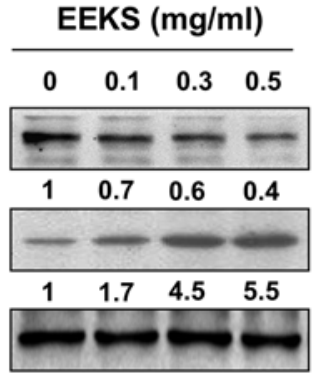

cytosolic

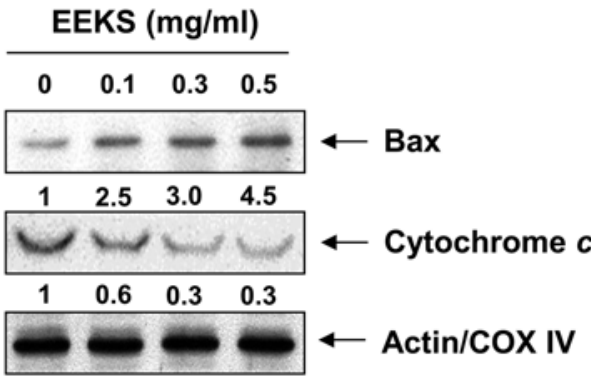

mitochondrial

C

\section{EEKS $(\mathrm{mg} / \mathrm{ml})$}

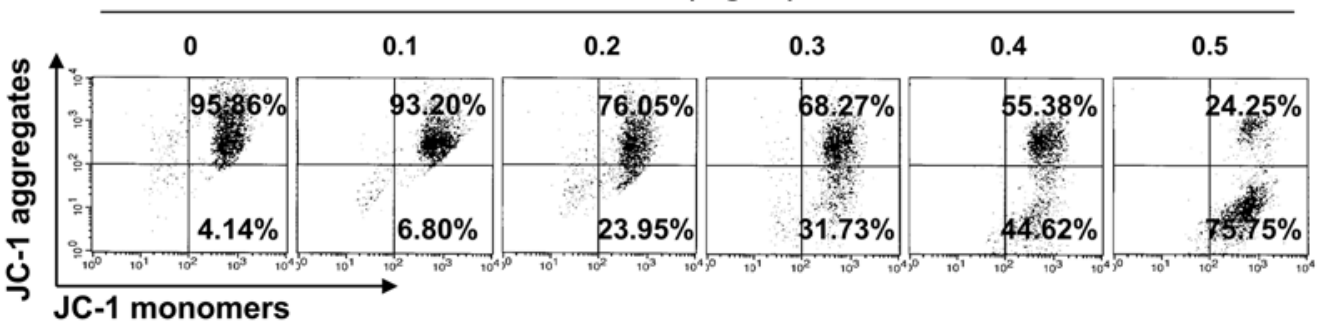

Figure 6. Effects of EEKS treatment on the levels of Bcl-2 family proteins and cytochrome $c$, and on the MMP values in HepG2 cells. The cells were treated with the indicated concentrations of EEKS for $24 \mathrm{~h}$. (A and B) The total cellular proteins (A) or cytosolic and mitochondrial proteins (B) were extracted and separated by SDS-polyacrylamide gel electrophoresis, followed by western blot analysis using the indicated antibodies and an enhanced chemiluminescence (ECL) detection system. Actin and cytochrome $c$ oxidase subunit 4 (COX IV) were used as internal controls for the cytosolic and mitochondrial fractions, respectively. The relative ratios of expression in the results of western blotting are presented at the bottom of each result as relative value of actin or COX IV expression. (C) The cells were collected and incubated with $10 \mu \mathrm{M} 5,5^{\prime}, 6,6^{\prime}$-tetrachloro-1,1'3,3'-tetraethyl-imidacarbocyanine iodide (JC-1) for 20 min at $37^{\circ} \mathrm{C}$ in the dark. The cells were then washed with PBS, and the mean JC-1 fluorescence intensity was detected using a flow cytometer. The data represent the means of two independent experiments. 

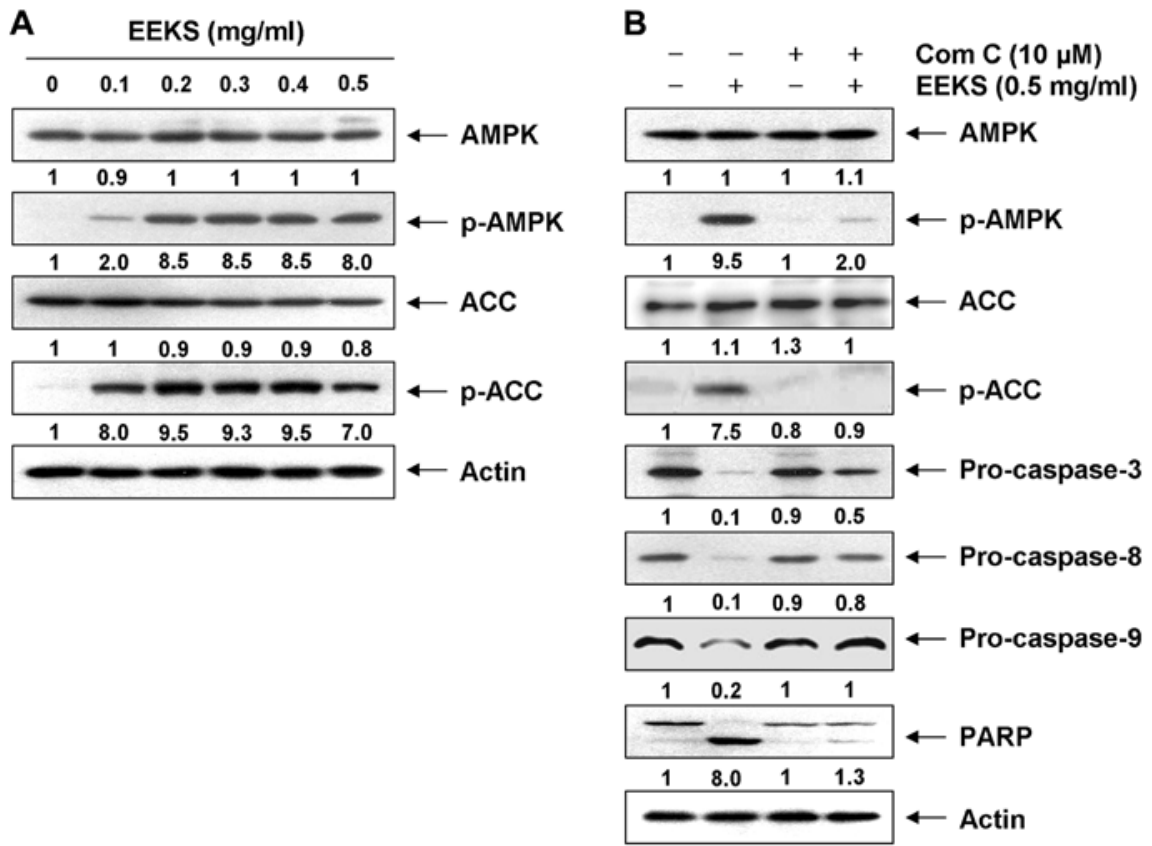

Figure 7. Activation of AMPK by EEKS treatment in HepG2 cells. The cells were treated with the indicated concentrations of EEKS for $24 \mathrm{~h}$ (A) or pre-treated with the AMPK inhibitor, compound C (Com C, $5 \mu \mathrm{M})$ for $1 \mathrm{~h}$ and then treated with EEKS $(0.5 \mathrm{mg} / \mathrm{ml})$ for $24 \mathrm{~h}(\mathrm{~B})$. Equal amounts of cell lysate were resolved by SDS-polyacrylamide gels, transferred to nitrocellulose membranes, and probed with the indicated antibodies. The proteins were visualized using an enhanced chemiluminescence (ECL) detection system. Actin was used as an internal control. The relative ratios of expression in the results of western blotting were presented at the bottom of each of the results as relative values of actin expression.

A

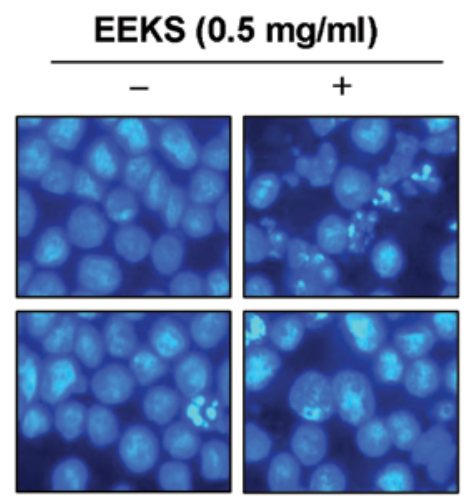

C

\section{EEKS $(0.5 \mathrm{mg} / \mathrm{ml})$}

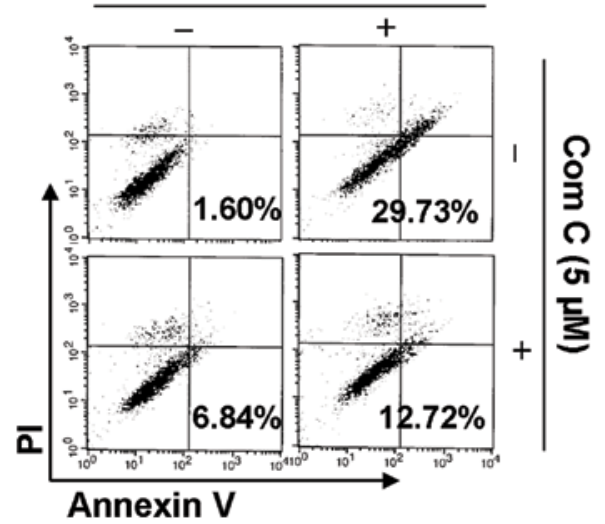

B

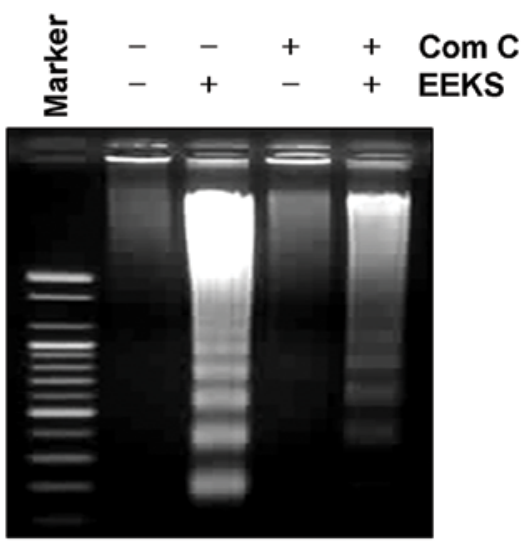

D

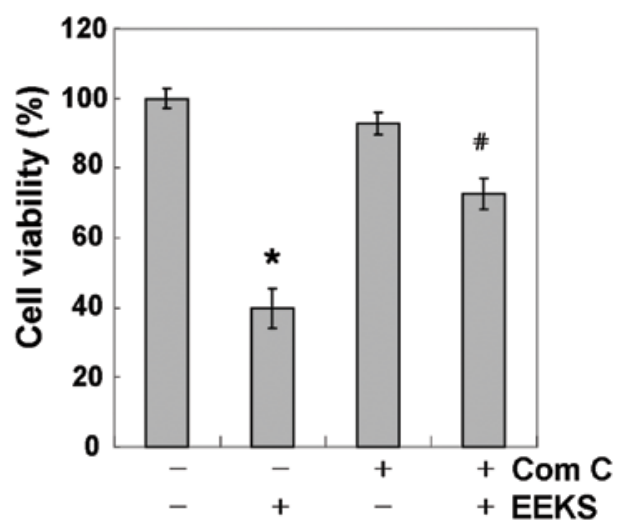

Figure 8. EEKS treatment triggered apoptosis through activation of AMPK in HepG2 cells. The cells were pre-treated with the AMPK inhibitor, compound C $(5 \mu \mathrm{M})$ for $1 \mathrm{~h}$, and then treated with EEKS $(0.5 \mathrm{mg} / \mathrm{ml})$ for $24 \mathrm{~h}$. (A) Nuclei were stained with DAPI solution and observed with a fluorescence microscope (original magnification, $\mathrm{x} 400$ ). (B) The genomic DNA from the cells was extracted, separated by agarose gel electrophoresis, and then visualized under UV light after staining with EtBr. (C) The percentage of apoptotic cells (Annexin $\mathrm{V}^{+}$cells) was analyzed using flow cytometry analysis. The data represent the means of the two different experiments. (D) Cell viability was determined by the MTT assay. Each point represents the mean \pm SD of three independent experiments ( ${ }^{*} \mathrm{p}<0.05$ vs. untreated control; ${ }^{\#} \mathrm{p}<0.05$ vs. EEKS-treated cells). 
in part, to the observed EEKS-induced apoptosis in HepG2 cells.

In addition to the extrinsic pathway, we also found that EEKS activated critical regulatory elements of the intrinsic mitochondrial executioner pathway, indicated by the loss of the MMP, translocation of Bax from the cytoplasm to mitochondria, release of cytochrome $c$ from mitochondria into the cytosol, and activation of caspase-9. These data suggest that Bax was oligomerized and anchored onto the outer mitochondrial membrane, thereby forming mitochondrial permeability transition pores that disrupted the MMP. Bax translocation could therefore be related to the mitochondrial response to the generation of $\mathrm{tBid}$, which led to mitochondrial disturbance, release of cytochrome $c$, and activation of caspase-9 to intensify the initial apoptotic response. However, pretreatment with the pan-caspase inhibitor, z-VAD-fmk, significantly attenuated EEKS-induced cell death and growth inhibition. Although the existence of a direct link between effects of EEKS on the intrinsic and extrinsic apoptosis pathways is unclear, our results indicate that EEKS appears to induce apoptosis in HepG2 cells primarily via a caspase-dependent action on both intrinsic and extrinsic apoptotic pathways.

Recently, activation of the AMPK signaling pathway has been shown to play a critical role in regulation of energy metabolism under both physiological and pathological conditions via downstream pathways relevant to the control of cellular proliferation $(16,17)$. AMPK is known to function as an intracellular energy sensor, being activated in response to an increased AMP/ATP ratio, a condition of energetic stress, to promote the catabolic pathway, thereby inhibiting cell proliferation $(18,19)$. Growing evidence suggests that AMPK is dysregulated in most cancer tissues, including HCC, when compared to normal tissues (26-28), and several studies have shown that AMPK activators exhibit inhibitory effects on HCC growth (21,26,27,29-31,36). Scientific interest continues to grow in the search for molecular pathways and novel compounds that target AMPK signaling as new potential therapeutic options for HCC. The results of the present study demonstrated that EEKS treatment of HepG2 cells promoted a concentration-dependent phosphorylation of AMPK and of ACC its downstream target.

We examined the action of EEKS on AMPK activation using the synthetic AMPK inhibitor compound C. As expected, the ability of EEKS to stimulate the phosphorylation of both AMPK and ACC was diminished markedly by compound $\mathrm{C}$ treatment, suggesting that EEKS could be an AMPK activator. Moreover, the inhibition of AMPK in HepG2 cells attenuated the EEKS-induced activation of caspases and prevented PARP cleavage. In addition, the induction of apoptosis and reduction in cell viability by EEKS was prevented by compound $\mathrm{C}$ pretreatment, strongly suggesting that EEKS activated AMPK, which led to caspase-dependent cell apoptosis in HepG2 cells.

In conclusion, the present study revealed that EEKS triggers apoptosis of human HCC HepG2 cells through activation of both the intrinsic caspase pathway and the extrinsic pathway. The resulting activation of caspases is responsible for the mediation of EEKS-induced apoptosis. In addition, we propose that EEKS-mediated apoptosis is caused by activation of AMPK, which may contribute to the induction of caspase- dependent HepG2 cell apoptosis. Our findings indicate that AMPK activation plays a pivotal role in EEKS-induced apoptosis of HepG2 cells, but further studies are needed to pinpoint the active compounds in the EEKS. Identification of the underlying molecular mechanisms and studies confirming the biological efficacy of EEKS using in vivo models are also required.

\section{Acknowledgements}

This study was supported by Basic Science Research Program through the National Research Foundation of Korea (NRF) grant funded by the Korea government (2013 041811, 2015R1A2A2A01004633 and 2014R1A1A1008460).

\section{References}

1. Kao JH and Chen DS: Changing disease burden of hepatocellular carcinoma in the Far East and Southeast Asia. Liver Int 25: 696-703, 2005

2. Venook AP, Papandreou C, Furuse J and de Guevara LL: The incidence and epidemiology of hepatocellular carcinoma: A global and regional perspective. Oncologist 15 (Suppl 4): 5-13, 2010.

3. Chen CJ, Wang LY, Lu SN, Wu MH, You SL, Zhang YJ, Wang LW and Santella RM: Elevated aflatox in exposure and increased risk of hepatocellular carcinoma. Hepatology 24: 38-42, 1996.

4. Feitelson MA, Sun B, Satiroglu Tufan NL, Liu J, Pan J and Lian Z: Genetic mechanisms of hepatocarcinogenesis. Oncogene 21: 2593-2604, 2002.

5. Thorgeirsson SS and Grisham JW: Molecular pathogenesis of human hepatocellular carcinoma. Nat Genet 31: 339-346, 2002.

6. Ben Ari Z, Weitzman E and Safran M: Oncogenic viruses and hepatocellular carcinoma. Clin Liver Dis 19: 341-360, 2015.

7. Avila MA, Berasain C, Sangro B and Prieto J: New therapies for hepatocellular carcinoma. Oncogene 25: 3866-3884, 2006.

8. Bruix J and Sherman M; American Association for the Study of Liver Diseases: Management of hepatocellular carcinoma: An update. Hepatology 53: 1020-1022, 2011.

9. Deng GL, Zeng S and Shen H: Chemotherapy and target therapy for hepatocellular carcinoma: New advances and challenges. World J Hepatol 7: 787-798, 2015.

10. Fadeel B and Orrenius S: Apoptosis: A basic biological phenomenon with wide-ranging implications in human disease. J Intern Med 258: 479-517, 2005.

11. Fulda $\mathrm{S}$ and Debatin KM: Extrinsic versus intrinsic apoptosis pathways in anticancer chemotherapy. Oncogene 25: 4798-4811, 2006.

12. Lavrik IN: Systems biology of apoptosis signaling networks. Curr Opin Biotechnol 21: 551-555, 2010.

13. Walczak H and Krammer PH: The CD95 (APO-1/Fas) and the TRAIL (APO-2L) apoptosis systems. Exp Cell Res 256: 58-66, 2000.

14. Ola MS, Nawaz M and Ahsan H: Role of Bcl-2 family proteins and caspases in the regulation of apoptosis. Mol Cell Biochem 351: 41-58, 2011.

15. Hensley P, Mishra M and Kyprianou N: Targeting caspases in cancer therapeutics. Biol Chem 394: 831-843, 2013.

16. Mantovani J and Roy R: Re-evaluating the general(ized) roles of AMPK in cellular metabolism. FEBS Lett 585: 967-972, 2011.

17. Li W, Saud SM, Young MR, Chen G and Hua B: Targeting AMPK for cancer prevention and treatment. Oncotarget 6: 7365-7378, 2015.

18. Marley AE, Sullivan JE, Carling D, Abbott WM, Smith GJ, Taylor IW, Carey F and Beri RK: Biochemical characterization and deletion analysis of recombinant human protein phosphatase 2C alpha. Biochem J 320: 801-806, 1996.

19. Oakhill JS, Scott JW and Kemp BE: AMPK functions as an adenylate charge-regulated protein kinase. Trends Endocrinol Metab 23: 125-132, 2012.

20. Hardie DG: AMPK: A key regulator of energy balance in the single cell and the whole organism. Int J Obes 32 (Suppl 4): S7-S12, 2008. 
21. Lee CW, Wong LL, Tse EY, Liu HF, Leong VY, Lee JM, Hardie DG, Ng IO and Ching YP: AMPK promotes p53 acetylation via phosphorylation and inactivation of SIRT1 in liver cancer cells. Cancer Res 72: 4394-4404, 2012.

22. Han JY, Park SH, Yang JH, Kim MG, Cho SS, Yoon G, Cheon SH and Ki SH: Licochalcone suppresses LXR $\alpha$-induced hepatic lipogenic gene expression through AMPK/Sirtl pathway activation. Toxicol Res 30: 19-25, 2014.

23. Hwang WC, Kim MK, Song JH, Choi KY and Min S: Inhibition of phospholipase D2 induces autophagy in colorectal cancer cells. Exp Mol Med 46: e124, 2014.

24. Min B, Lee H, Song JH, Han MJ and Chung J: Arctiin inhibits adipogenesis in 3T3-L1 cells and decreases adiposity and body weight in mice fed a high-fat diet. Nutr Res Pract 8: 655-661, 2014.

25. Rehman G, Shehzad A, Khan AL and Hamayun M: Role of AMP-activated protein kinase in cancer therapy. Arch Pharm (Weinheim) 347: 457-468, 2014.

26. Martelli AM, Chiarini F, Evangelisti C, Ognibene A, Bressanin D, Billi AM, Manzoli L, Cappellini A and McCubrey JA: Targeting the liver kinase B1/AMP-activated protein kinase pathway as a therapeutic strategy for hematological malignancies. Expert Opin Ther Targets 16: 729-742, 2012.

27. Zheng L, Yang W, Wu F, Wang C, Yu L, Tang L, Qiu B, Li Y, Guo L, Wu M, et al: Prognostic significance of AMPK activation and therapeutic effects of metformin in hepatocellular carcinoma. Clin Cancer Res 19: 5372-5380, 2013.

28. Lv Q, Zhen Q, Liu L, Gao R, Yang S, Zhou H, Goswami R and Li Q: AMP-kinase pathway is involved in tumor necrosis factor alpha-induced lipid accumulation in human hepatoma cells. Life Sci 131: 23-29, 2015.

29. Yu R, Zhang ZQ, Wang B, Jiang HX, Cheng L and Shen LM: Berberine-induced apoptotic and autophagic death of HepG2 cells requires AMPK activation. Cancer Cell Int 14: 49, 2014

30. Ishijima N, Kanki K, Shimizu H and Shiota G: Activation of AMP-activated protein kinase by retinoic acid sensitizes hepatocellular carcinoma cells to apoptosis induced by sorafenib. Cancer Sci 106: 567-575, 2015.

31. Wu JJ, Omar HA, Lee YR, Teng YN, Chen PS, Chen YC, Huang HS, Lee KH and Hung JH: 6-Shogaol induces cell cycle arrest and apoptosis in human hepatoma cells through pleiotropic mechanisms. Eur J Pharmacol 762: 449-458, 2015.

32. Rattan R, Giri S, Singh AK and Singh I: 5-Aminoimidazole4-carboxamide-1-beta-D-ribofuranoside inhibits cancer cell proliferation in vitro and in vivo via AMP-activated protein kinase. J Biol Chem 280: 39582-39593, 2005

33. Zhao H, Zhu H, Lin Z, Lin G and Lv G: Compound 13, an a1-selective small molecule activator of AMPK, inhibits Helicobacter pylori-induced oxidative stresses and gastric epithelial cell apoptosis. Biochem Biophys Res Commun 463: 510-517, 2015

34. Qu Z, Zhang Y, Liao M, Chen Y, Zhao J and Pan Y: In vitro and in vivo antitumoral action of metformin on hepatocellular carcinoma. Hepatol Res 42: 922-933, 2012.

35. Saito T, Chiba T, Yuki K,Zen Y,Oshima M, Koide S, Motoyama T, Ogasawara S, Suzuki E, Ooka Y, et al: Metformin, a diabetes drug, eliminates tumor-initiating hepatocellular carcinoma cells. PLoS One 8: e70010, 2013.

36. Yang X, Sun D, Tian Y, Ling S and Wang L: Metformin sensitizes hepatocellular carcinoma to arsenic trioxide-induced apoptosis by downregulating Bcl2 expression. Tumour Biol 36: 2957-2964, 2015.
37. Chou CW, Cheng YW and Tsai $\mathrm{CH}$ : Phyllostachys edulis extract induces apoptosis signaling in osteosarcoma cells, associated with AMPK activation. Drug Des Devel Ther 8: 1577-1584, 2014.

38. Lee HW, Jang KS, Choi HJ, Jo A, Cheong JH and Chun KH: Celastrol inhibits gastric cancer growth by induction of apoptosis and autophagy. BMB Rep 47: 697-702, 2014.

39. Shin JA, Kwon KH and Cho SD: AMPK-activated protein kinase activation by Impatiens balsamina L. is related to apoptosis in HSC-2 human oral cancer cells. Pharmacogn Mag 11: 136-142, 2015.

40. Yang TP, Lee HJ, Ou TT, Chang YJ and Wang CJ: Mulberry leaf polyphenol extract induced apoptosis involving regulation of adenosine monophosphate-activated protein kinase/fatty acid synthase in a p53-negative hepatocellular carcinoma cell. J Agric Food Chem 60: 6891-6898, 2012

41. Yang G, Li X, Li X, Wang L, Li J, Song X, Chen J, Guo Y, Sun X, Wang S, et al: Traditional Chinese medicine in cancer care: A review of case series published in the chinese literature. Evid Based Complement Alternat Med 2012: 751046, 2012

42. Wang X, Feng Y, Wang N, Cheung F, Tan HY, Zhong S, Li C and Kobayashi S: Chinese medicines induce cell death: The molecular and cellular mechanisms for cancer therapy. Biomed Res Int 2014: 530342, 2014

43. Shin JS, Kim YM, Hong SS, Kang HS, Yang YJ, Lee DK, Hwang BY, Ro JS and Lee MK: Induction of neurite outgrowth by (-)-(7R, 8S)-dihydrodehydrodiconiferyl alcohol from PC12 cells. Arch Pharm Res 28: 1337-1340, 2005.

44. Jun DH, Lee JT, Cheon SJ, Lee CE, Kim TH, Lee DH, Han J and Kim SH: Polyphenol and anti-oxidant effects of Kalopanax septemlobus Koidz. leaf extracts. Korean J Plant Res 22: 343-348, 2009.

45. Wang LS, Zhao DQ, Xu TH, Zhou XF, Yang XW and Liu YH: A new triterpene hexaglycoside from the bark of Kalopanax septemlobus (Thunb.) Koidz. Molecules 14: 4497-4504, 2009.

46. Kim HJ, Kim MJ, Oh SI, Hwangbo MH, Jang SJ, Kim HI and Lee IS: Antioxidant activity of Kalopanax pictus leaf extract and its effects on the quality characteristics of fried pork skin. Korean J Food Sci Technol 44: 185-190, 2012.

47. Duriez PJ and Shah GM: Cleavage of poly(ADP-ribose) polymerase: A sensitive parameter to study cell death. Biochem Cell Biol 75: 337-349, 1997.

48. de Graaf AO, de Witte T and Jansen JH: Inhibitor of apoptosis proteins: New therapeutic targets in hematological cancer? Leukemia 18: 1751-1759, 2004.

49. Danson S, Dean E, Dive C and Ranson M: IAPs as a target for anticancer therapy. Curr Cancer Drug Targets 7: 785-794, 2007.

50. Scorrano L and Korsmeyer SJ: Mechanisms of cytochrome $c$ release by proapoptotic BCL-2 family members. Biochem Biophys Res Commun 304: 437-444, 2003.

51. Jourdain A and Martinou JC: Mitochondrial outer-membrane permeabilization and remodelling in apoptosis. Int J Biochem Cell Biol 41: 1884-1889, 2009.

52. Scott JW, Norman DG, Hawley SA, Kontogiannis L and Hardie DG: Protein kinase substrate recognition studied using the recombinant catalytic domain of AMP-activated protein kinase and a model substrate. J Mol Biol 317: 309-323, 2002.

53. Hassan M, Watari H, AbuAlmaaty A, Ohba Y and Sakuragi N: Apoptosis and molecular targeting therapy in cancer. BioMed Res Int 2014: 150845, 2014

54. Kantari $\mathrm{C}$ and Walczak H: Caspase- 8 and bid: Caught in the act between death receptors and mitochondria. Biochim Biophys Acta 1813: 558-563, 2011. 